\title{
Surgical management of cavernous malformations presenting with drug-resistant epilepsy
}

\section{Mario Arturo Alonso-Vanegas ${ }^{1}{ }^{*}$, José M. Cisneros-Franco ${ }^{1}$ and Taisuke Otsuki ${ }^{2}$}

1 Instituto Nacional de Neurología y Neurocirugía "Manuel Velasco Suárez," Mexico City, Mexico

${ }^{2}$ National Center of Neurology and Psychiatry, Tokyo, Japan

\section{Edited by:}

Fernando Cendes, University of

Campinas, Brazil

Reviewed by:

Marco T. Medina, Universidad

Nacional Autónoma de Honduras,

Honduras

Nigel C. K. Tan, National

Neuroscience Institute, Singapore

*Correspondence:

Mario Arturo Alonso-Vanegas,

Programa Prioritario de Epilepsia,

Instituto Nacional de Neurología y Neurocirugía, Insurgentes sur 3877

col. La Fama, Tlalpan, México D.F. CP

14269, México.

e-mail: alonsomario@hotmail.com
Cerebral cavernous malformations (CMs) are dynamic lesions characterized by continuous size changes and repeated bleeding. When involving cortical tissue, CMs pose a significant risk for the development of drug-resistant epilepsy, which is thought to be result of an altered neuronal network caused by the lesion itself and its blood degradation products. Preoperative evaluation should comprise a complete seizure history, neurological examination, epilepsy-oriented MRI, EEG, video-EEG, completed with SPECT, PET, functional $\mathrm{MRI}$, and/or invasive monitoring as needed. Radiosurgery shows variable rates of seizure freedom and a high incidence of complications, thus microsurgical resection remains the optimal treatment for CMs presenting with drug-resistant epilepsy. Two thirds of patients reach Engel I class at 3-year follow-up, regardless of lobar location. Those with secondarily generalized seizures, a higher seizure frequency, and generalized abnormalities on preoperative or postoperative EEG, show poorer outcomes, while factors such as gender, duration of epilepsy, lesion size, age, bleeding at the time of surgery, do not correlate consistently with seizure outcome. Electrocorticography and a meticulous removal of all cortical hemosiderin - beyond pure lesionectomy - reduce the risk of symptomatic recurrences.

Keywords: epilepsy surgery, cavernous malformation, drug-resistant epilepsy, electrocorticography, partial onset epilepsy

\section{INTRODUCTION}

Cerebral cavernous malformations (CMs), also known as cavernous angiomas, cavernomas, or cavernous hemangiomas, were first described in the central nervous system by Virchow in 1863 (Iza-Vallejo et al., 2005) and characterized microscopically by Mccormick (1966). These mulberry-like lesion consist of intertwined clusters of thin-walled vascular sinusoids lined by a thin endothelium lacking smooth muscle, elastin, and intervening parenchyma, surrounded by hemosiderin deposits and gliosis (Raychaudhuri et al., 2005). CMs account for about 10\% of all symptomatic vascular malformations, being the most frequent angiographically occult intracranial vascular malformations (Gomori et al., 1986), with an estimated frequency of $0.4-0.9 \%$ (Otten et al., 1989; Brown et al., 2005), and an annual bleeding rate laying somewhere between 0.7 and $1.4 \% /$ lesion/year, or 0.25-3.1\%/person/year of exposure (Kim et al., 1997; Stefan and Hammen, 2004; Ferroli et al., 2006).

Their occurrence can be sporadic, or as an autosomal dominant condition with variable expression and incomplete penetrance. The first recognized gene association (CCM1), was mapped in the long arm of chromosome 7 ( $7 q 11$ to $t q 12)$, upon studying the familial form of CM in a Hispanic family (Dubovsky et al., 1995). Three genes implied in the pathogenesis have been identified, all of them expressed in neurons rather than in blood vessels, suggesting an impaired signaling between these structures (Revencu and Vikkula, 2006).

It is well recognized the changing nature of CMs. This dynamism is determined by both extrinsic - hemorrhage, calcifications, thrombosis, and intrinsic factors - pseudotumoral growth of the cavernous matrix - that result in growing or shrinking of established malformation; and by pathologic angiogenic factors in de novo appearing lesions (Houtteville, 1997).

\section{CMS AND EPILEPSY}

Clinical manifestations, when present, are variable and include headache, seizures, hemorrhage, and sudden onset focal neurological deficits (FND; Raychaudhuri et al., 2005). The latter are the most common presentation of brainstem lesions, whereas seizures are the most frequent manifestation of supratentorial lesions (Awad and Jabbour, 2006; Van Gompel et al., 2010).

Epileptic seizures linked to CMs are often drug-resistant (Robinson et al., 1991; Kim et al., 1997). Cortical involvement is the most relevant risk factor for epilepsy. A greater diameter, the absence of edema, and localization in the left hemisphere are also associated with the occurrence of epilepsy. Mesiotemporal archicortical CMs pose a significantly higher risk for the development of epilepsy than neocortical lesions, a group that shows no differences regarding lobar location (Yeon et al., 2009; Menzler et al., 2010). Patients with peri-limbic or anterior temporal lesions may show signs of dual pathology, consistent in macroscopically-hippocampal sclerosis - microscopically, or only functional abnormalities within the mesial temporal lobe (Cendes et al., 1995; Stefan and Hammen, 2004).

The mass effect does not completely account for CMs' high epileptogenicity, as the clinician may appreciate when dealing with larger lesions, namely other kinds of AVMs - in the absence of 
prior hemorrhage or FND - and gliomas, which are less likely to present with seizures (Awad et al., 1991; Josephson et al., 2011). This feature, referred to as secondary epileptogenesis (Morrell, 1985), has been studied in CMs. Williamson et al. (2003) performed intracellular recordings from neurons adjacent to gliomas and cavernomas, finding that neurons adjacent to CMs were more likely to show large complex spontaneous synaptic events than neurons adjacent to tumors. These neurons also showed more pronounced responses to synaptic stimulation, e.g., multiple action potentials riding on prolonged excitatory postsynaptic potentials were evoked in the majority of these cells, as opposed to those belonging to the tumor group (Williamson et al., 2003).

Intralesional bleeding is another factor to consider. This event may partially destroy the CM, leaving residual cysts, and calcifications (Pozzati et al., 1989). It is thought that the breakdown of blood products results in gradual deposition of hemosiderin and hemin in the cerebral tissue surrounding the malformation (Kim et al., 1997); ultimately leading to biochemical abnormalities that become more prevalent and significant as the duration of epilepsy continues. The latter might explain why a longer history of epilepsy is a predictive of poorer results following pure lesionectomy, i.e., restricted surgical removal of the $\mathrm{CM}$, respecting the hemosiderinstained gliotic perilesional tissue (Ferroli et al., 2006). In addition, reactive glial proliferation may be epileptogenic.

\section{PREOPERATIVE EVALUATION}

A detailed preoperative epileptological and radiological work-up tailored to the individual's pathology allows the clinician to classify seizures according to the ILAE guidelines. Clinical history, neurological examination, epilepsy-oriented MRI, scalp EEG, and video-EEG are essential (Von Der Brelie and Schramm, 2011). Spin echo and gradient echo sequences using T1 and T2-weighed MRI provide useful information on the CM hemodynamics; and also permit to classify them in four types according to the findings focused on signal characteristics (Zabramski et al., 1994).

Complementary studies include interictal and/or ictal SPECT, PET, functional MRI, magnetoencephalography (Stefan et al., 2004), intracarotid amobarbital testing (Stefan et al., 2004; Yeon et al., 2009), electrocorticography (ECoG), or implantation of intracerebral or subdural grid electrodes when needed (Siegel et al., 2000; Ferrier et al., 2007). Computer tomography with contrast enhancement (Stavrou et al., 2008) or angiography can also be used, although the latter usually fails to identify CMs (Gomori et al., 1986; Stavrou et al., 2008).

\section{MANAGEMENT}

Radiosurgery leads to the progressive obliteration of CMs by endothelial cell proliferation, with consequent luminal closure. This process takes 1-3 years on average to complete (Schneider et al., 1997), a period of time during which the risk of hemorrhage remains. Moreover, serious complications are not uncommon, with as much as $41 \%$ of patients presenting neurological deterioration after treatment and $27 \%$ requiring microsurgical extirpation (Karlsson et al., 1998). In fact, pathological studies on previously radiated patients undergoing surgery show that radiation does not always warrant complete vascular obliteration (Gewirtz et al., 1998).
Although seizure freedom is reported to reach 52\% (Regis et al., 2000), there is still a lack of consensus on the indications for radiosurgery and the appropriate dose of irradiation, let alone the limited evidence supporting a protective role against rebleeding (Kim et al., 1997; Pollock, 2008). For these reasons, excision remains the optimal therapeutic strategy for patients with drug-resistant epilepsy. Standard microsurgical technique is widely accepted, and variations in surgical behavior exist among different groups. For instance, some of them describe the routine (Ferroli et al., 2006) or occasional (Kivelev et al., 2011) use of a stereotactic device or frameless neuronavigation system. A mini-invasive transulcal approach has been described (Ferroli et al., 2006); alternatively, a transsylvian approach may be useful for anteromedial temporal $\mathrm{CMs}$, or transcortical excision, at times using intersulcal dissection, for other locations (Kivelev et al., 2011). Neurophysiologic monitoring, in the form of direct cortical mapping and monitoring of neurological functions are used for CMs in eloquent locations (Ferroli et al., 2006).

The amount of resected tissue is also a matter of discussion. For instance, Yeon et al. (2009) take into account seizure frequency, opting for more aggressive approaches for drug-resistant epilepsy, i.e., extended rather than simple lesionectomy in patients without mesial temporal lesions; and tailored resection rather than standard temporal lobectomy for mesiotemporal CMs (Yeon et al., 2009). On the other hand, Ferroli et al. (2006) endorse a two-step surgical policy for patients with epilepsy secondary to CMs, attempting at first a pure lesionectomy, followed by invasive localization and tailored removal of the epileptogenic zone in those patients with drug-resistant seizures at 1-2 years follow-up (Ferroli et al., 2006).

\section{GENERAL OUTCOME}

Up to $17 \%$ of patients may develop neurological symptoms (sensorimotor deficits and homonymous hemi or quadrantanopsia) immediately after the operation (Ferroli et al., 2006; Stavrou et al., 2008). Nonetheless, at follow-up, the rate of neurological deficits including severe headache, slight dysphasia, sensory disturbances, ataxia, severe hemiparesis, pontocerebellar degeneration, descends to $2.6-8 \%$ (Zevgaridis et al., 1996; Baumann et al., 2006; Ferroli et al., 2006; Stavrou et al., 2008; Kivelev et al., 2011). No mortality related to the procedure is reported.

Data is still limited regarding neuropsychological outcome. In one study, $15 \%$ of patients complained of postoperative memory deficits, the half of these presented with only temporary shortterm memory decline. The rest underwent neuropsychological evaluation, reporting new memory deficit $(4 \%)$ or worsening of previous symptoms (4\%). Postoperative depression and fatigue were also assessed, finding a $9.4 \%$ rate of new-onset symptoms (Kivelev et al., 2011).

\section{SEIZURE OUTCOME}

It is not easy to systematically evaluate seizure outcome due to the following limitations: several studies include patients whose main complaint was not epilepsy; definitions for intractability are not always clear; group subdivision is not uniform among studies regarding the evolution, frequency, and/or severity of seizures; control groups are compared against lesionectomy or epilepsy 
surgery, but rarely both. Finally, standardized classifications Engel et al. (1993), ILAE (Wieser et al., 2001) - are yet to be universally adopted.

\section{OVERALL SEIZURE OUTCOME}

The largest series to date, including 168 patients, showed an Engel I rate of $70 \%$ ( $48 \%$ IA) after 1 year. This figure declined to 68 and $65 \%$ for the second and third, respectively (Baumann et al., 2007), a phenomenon previously described by Kim et al. (1997), arguably due to the lack of follow-up for patients who became seizure-free. In contrast, smaller series report up to $82-84 \%$ seizure freedom rates (Casazza et al., 1996; Cappabianca et al., 1997).

\section{LENGTH OF FOLLOW-UP}

As commented above, slight differences on outcome are to be expected as follow-up lengthens, namely after the third year (Baumann et al., 2007; Von Der Brelie and Schramm, 2011), while this is not a constant finding (Yeon et al., 2009).

\section{LOBAR LOCATION}

There seems not to be any correlation between the lobar location nor the side of CMs and seizure freedom (Cappabianca et al., 1997; Baumann et al., 2007). At 1-year follow-up, the outcome was better for patients with mesiotemporal cavernomas and worst for neocortical locations (Baumann et al., 2007), but subsequent comparisons showed no differences.

Lesion location within the temporal lobe does not correlate with epilepsy outcome, i.e., patients with mesial or lateral lesions have the same chance of having a favorable seizure outcome (Yeon et al., 2009; Kivelev et al., 2011).

\section{SIZE OF LESION}

A size of less than $1.5 \mathrm{~cm}$ diameter has been associated with better seizure control during the first 2 years but no differences arise at longer evaluation (Baumann et al., 2007; Englot et al., 2011), a finding confirmed later on (Yeon et al., 2009; Englot et al., 2011).

\section{TYPE OF SEIZURES}

Patients with simple partial and complex partial seizures are more likely to become asymptomatic than patients affected by secondarily generalized seizures, although this analysis is not routinely performed by most of studies (Baumann et al., 2007; Englot et al., 2011).

\section{DURATION OF EPILEPSY}

The theory of secondary epileptogenesis states that a prolonged preoperative history of epilepsy brings an increased risk for worse seizure outcome as a result of developing remote epileptogenic foci (Morrell, 1985; Cohen et al., 1995; Cappabianca et al., 1997). The impact of this variable is controversial, for there are series that correlate the duration of epilepsy with seizure outcome, i.e., they report a slightly (Stavrou et al., 2008) or significantly (Cohen et al., 1995; Casazza et al., 1996; Zevgaridis et al., 1996; Cappabianca et al., 1997; Schroeder et al., 1997; Moran et al., 1999; Hammen et al., 2007; Englot et al., 2011) poorer outcome for patients with more than 1-2 years of seizure history, with the notable exception of patients with sporadic seizures over a long period of time. On the other hand, others report similar results for patients with 0.5 to more than 10 years of seizure history (Baumann et al., 2007; Kivelev et al., 2011).

Yeon et al. (2009) did a sub-analysis within their nonlesionectomy group, for which duration of illness showed statistical significance only when Engel class IA was set aside and compared to the other classes considered as a whole (Yeon et al., 2009).

\section{GENDER}

Gender differences on outcome, when present, show that men seem to have a higher chance to become seizure-free (Cohen et al., 1995; Cappabianca et al., 1997; Stavrou et al., 2008), but, once again, it is not a constant finding (Baumann et al., 2007; Yeon et al., 2009).

\section{AGE}

Some studies show a better outcome in patients whose first seizure occurred after age 30 (Cohen et al., 1995; Baumann et al., 2007) or 40 (Cappabianca et al., 1997). In contrast, others considered age at onset to be irrelevant (Moran et al., 1999; Stavrou et al., 2008; Yeon et al., 2009). Baumann et al. (2007) found better seizure control for patients aged 30 or more at the time of surgery.

\section{PREOPERATIVE EEG}

There is a reported correlation between epileptiform abnormalities after the first unprovoked seizure and seizure recurrence (Van Donselaar et al., 1992). Subjects with normal findings on preoperative scalp EEG have better odds of being seizure free (Kivelev et al., 2011), as opposed to patients with multifocal epileptic activity (Baumann et al., 2007).

\section{POSTOPERATIVE EEG}

Kivelev et al. (2011) found no correlation between neurophysiological evaluation and epilepsy control, but an earlier study described an association of interictal epileptiform activity and seizure persistence (Di Gennaro et al., 2004).

\section{LESION BLEEDING AT THE TIME OF SURGERY}

Perilesional bleeding - old or recent - whether documented on MRI or directly observed during surgery, did not affect outcome on Baumann et al. (2007) study, but was considered as negative prognostic factor by Stefan et al. (2004) and Stefan and Hammen (2004).

\section{FREQUENCY AND NUMBER OF SEIZURES}

In two studies patients were classified according to the total number of seizures in three groups, i.e., $1,2-5$, and $>5$ seizures, achieving a seizure-free rate of $100,69-100$, and $62.5-69 \%$, respectively (Cappabianca et al., 1997; Kivelev et al., 2011).

A high seizure frequency before surgery has been shown to worsen the postoperative outcome in some series (Cohen et al., 1995; Cappabianca et al., 1997; Moran et al., 1999; Stefan and Hammen, 2004; Ferroli et al., 2006; Englot et al., 2011) but had no effect in others (Casazza et al., 1996; Baumann et al., 2007; Stavrou et al., 2008). In their study, Yeon et al. (2009) named "intractable" those patients presenting $>1$ monthly seizure over a 1 -year period. 
Seventy-two percent of these patients were in Engel I class (54.5\% IA), as opposed to $89.5 \%$ (84.2\% IA) of subjects with sporadic seizures (Yeon et al., 2009).

\section{THE ROLE OF "PURE" LESIONECTOMY}

The extension of excision is subject of controversy. Several studies report a better outcome when surrounding gliosis and hemosiderin are removed (Piepgras et al., 1993; Yeh et al., 1993; Cohen et al., 1995; Casazza et al., 1997; Kim et al., 1997; Siegel et al., 2000; Stefan and Hammen, 2004; Baumann et al., 2006; Hammen et al., 2007; Stavrou et al., 2008; Menzler et al., 2010), while others fail to find significant differences (Casazza et al., 1996; Zevgaridis et al., 1996; Cappabianca et al., 1997).

The problem arises when trying to compare techniques due to the lack of a control group - one of medical treatment - that can be compared with both lesionectomy and more extended resections in the same study. For instance, Yeon et al. (2009) found no difference between lesionectomy and other methods; but the decision of what procedure to perform was predetermined by their protocol, which, in turn favors the use of a more aggressive approach for patients with intractable epilepsy, a group in which blood degradation products are thought to play a major role in the aforementioned mechanisms of seizure propagation and secondary epileptogenesis (Morrell, 1991; Yeh and Privitera, 1991).

Lesionectomy alone appears to be beneficial for patients with sporadic seizures or evolution shorter than 1 year (Acciarri et al., 1995; Cohen et al., 1995; Casazza et al., 1996; Zevgaridis et al., 1996; Cappabianca et al., 1997; Ferroli et al., 2006). In this subset of individuals, it has been suggested to reserve a more invasive approach for persisting or relapsing cases (Yeon et al., 2009).

Growing evidence both in clinical and basic grounds suggests that resection of all cortical hemosiderin might improve seizure outcome, whereas incomplete removal of subcortical hemosiderin to spare eloquent tracts might not influence postoperative seizures (Siegel et al., 2000; Baumann et al., 2006; Menzler et al., 2010).

\section{REFERENCES}

Acciarri, N., Giulioni, M., Padovani, R., Galassi, E., and Gaist, G. (1995). Surgical management of cerebral cavernous angiomas causing epilepsy. J. Neurosurg. Sci. 39, 13-20.

Awad, I., and Jabbour, P. (2006). Cerebral cavernous malformations and epilepsy. Neurosurg. Focus 21, e7.

Awad, I. A., Rosenfeld, J., Ahl, J., Hahn, J. F., and Luders, H. (1991). Intractable epilepsy and structural lesions of the brain: mapping, resection strategies, and seizure outcome. Epilepsia 32, 179-186.

Baumann, C. R., Acciarri, N., Bertalanffy, H., Devinsky, O., Elger, C. E., Lo Russo, G., Cossu, M., Sure, U., Singh, A., Stefan, H., Hammen, T., Georgiadis, D., Baumgartner, R. W., Andermann, F., and Siegel, A. M. (2007). Seizure

In particular, transoperative ECoG-guided resection is associated with more extensive resections and, more importantly, is a good prognostic factor for seizure freedom when compared to patients without ECoG (Sugano et al., 2007; Komotar et al., 2008; Van Gompel et al., 2009).

\section{CONCLUSION}

Comprehensive research is still needed to reach fully understanding of CMs on the molecular level and its implications on clinical grounds (Raychaudhuri et al., 2005).

Early microsurgical resection is an effective and safe therapy for patients with CMs and symptomatic epilepsy, let alone CMs' well known inherent risk of bleeding. For this purpose, the seizures must be carefully classified; the site of the lesion and the presence/absence of dual pathology accurately determined; and the focal epileptic activity, the risk of bleeding, and neuropsychological status carefully evaluated (Stefan and Hammen, 2004).

It is strongly recommended the complete lesion removal including the neighboring epileptogenic brain tissue for drug-resistant epilepsy, performing transoperative neurophysiological monitoring $(\mathrm{ECoG})$, because subtotal removal of a $\mathrm{CM}$ is associated with a high risk of symptomatic recurrences (Kim et al., 1997; Baumann et al., 2006; Hammen et al., 2007; Englot et al., 2011).

Published case series and reviews emphasize the need of further prospective studies with homogeneous inclusion criteria with the purpose of generating comparable data (Von Der Brelie and Schramm, 2011). In this spirit, we suggest that upcoming studies adhere to the revised definition of drug-resistance (Kwan et al., 2010), state clearly the demographics of the group studied, the type and number of seizures, illness duration, history of bleeding, neurological examination, paraclinical findings - MRI, EEG, videoEEG, and, when suitable, metabolic and/or functional studies, size, location, surgical approach, resection type, transoperatory clinical and paraclinical findings, transient and permanent neurological deficits, the use of Engel and/or ILAE outcome classifications, and the length of follow-up.

and epilepsy: seizure outcome after lesionectomy on a series of 35 patients. Clin. Neurol. Neurosurg. 99, 179-183.

Casazza, M., Avanzini, G., Ciceri, E., Spreafico, R., and Broggi, G. (1997). Lesionectomy in epileptogenic temporal lobe lesions: preoperative seizure course and postoperative outcome. Acta Neurochir. Suppl. 68, 64-69.

Casazza, M., Broggi, G., Franzini, A., Avanzini, G., Spreafico, R., Bracchi, M., and Valentini, M. C. (1996). Supratentorial cavernous angiomas and epileptic seizures: preoperative course and postoperative outcome. Neurosurgery 39, 26-32; discussion 32-24.

Cendes, F., Cook, M. J., Watson, C., Andermann, F., Fish, D. R., Shorvon, S. D., Bergin, P., Free, S., Dubeau, F., and Arnold, D. L. (1995). Frequency and characteristics of dual pathology in patients with lesional epilepsy. Neurology 45, 2058-2064.

Cohen, D. S., Zubay, G. P., and Goodman, R. R. (1995). Seizure outcome after lesionectomy for cavernous malformations. J. Neurosurg. 83, 237-242.

Di Gennaro, G., Quarato, P. P., Sebastiano, F., Esposito, V., Onorati, P., Mascia, A., Romanelli, P., Grammaldo, L. G., Falco, C., Scoppetta, C., Eusebi, F., Manfredi, M., and Cantore, G. (2004). Postoperative EEG and seizure outcome in temporal lobe epilepsy surgery. Clin. Neurophysiol. 115, 1212-1219.

Dubovsky, J., Zabramski, J. M., Kurth, J., Spetzler, R. F., Rich, S. S., Orr, H. T., and Weber, J. L. (1995). A gene responsible for cavernous malformations of the brain maps to chromosome 7q. Hum. Mol. Genet. 4, 453-458. 
Engel, J., Van Ness, P. C., Rasmussen, T. C., and Ojemann, L. M. (1993). "Outcome with respect to epileptic seizures," in Surgical Treatment of the Epilepsies, ed. J. Engel (New York: Raven Press), 609-621.

Englot, D. J., Han, S. J., Lawton, M. T., and Chang, E. F. (2011). Predictors of seizure freedom in the surgical treatment of supratentorial cavernous malformations. J. Neurosurg. 115, 240-244.

Ferrier, C. H., Aronica, E., Leijten, F. S., Spliet, W. G., Boer, K., Van Rijen, P. C., and Van Huffelen, A. C. (2007). Electrocorticography discharge patterns in patients with a cavernous hemangioma and pharmacoresistant epilepsy. J. Neurosurg. 107, 495-503.

Ferroli, P., Casazza, M., Marras, C., Mendola, C., Franzini, A., and Broggi, G. (2006). Cerebral cavernomas and seizures: a retrospective study on 163 patients who underwent pure lesionectomy. Neurol. Sci. 26, 390-394.

Gewirtz, R. J., Steinberg, G. K., Crowley, R., and Levy, R. P. (1998). Pathological changes in surgically resected angiographically occult vascular malformations after radiation. Neurosurgery 42, 738-742; discussion 742-733.

Gomori, J. M., Grossman, R. I., Goldberg, H. I., Hackney, D. B., Zimmerman, R. A., and Bilaniuk, L. T. (1986). Occult cerebral vascular malformations: high-field MR imaging. Radiology 158, 707-713.

Hammen, T., Romstock, J., Dorfler, A., Kerling, F., Buchfelder, M., and Stefan, H. (2007). Prediction of postoperative outcome with special respect to removal of hemosiderin fringe: a study in patients with cavernous haemangiomas associated with symptomatic epilepsy. Seizure 16, 248-253.

Houtteville, J. P. (1997). Brain cavernoma: a dynamic lesion. Surg. Neurol. 48, 610-614.

Iza-Vallejo, B., Mateo-Sierra, O., Mosqueira-Centurion, B., RuizJuretschke, F., and Carrillo, R. (2005). Cerebral cavernomas. A review and update of aetiological, clinical and therapeutic features. Rev. Neurol. 41, 725-732.

Josephson, C. B., Leach, J. P., Duncan, R., Roberts, R. C., Counsell, C. E., and Al-Shahi Salman, R. (2011). Seizure risk from cavernous or arteriovenous malformations: prospective population-based study. Neurology 76, 1548-1554.

Karlsson, B., Kihlstrom, L., Lindquist, C., Ericson, K., and Steiner, L.
(1998). Radiosurgery for cavernous malformations. J. Neurosurg. 88, 293-297.

Kim, D. S., Park, Y. G., Choi, J. U., Chung, S. S., and Lee, K. C. (1997). An analysis of the natural history of cavernous malformations. Surg. Neurol. 48, 9-17; discussion 17-18.

Kivelev, J., Niemela, M., Blomstedt, G., Roivainen, R., Lehecka, M., and Hernesniemi, J. (2011). Microsurgical treatment of temporal lobe cavernomas. Acta Neurochir. (Wien) 153, 261-270.

Komotar, R. J., Mikell, C. B., and Mckhann, G. M. II. (2008). "Epilepsy surgery" versus lesionectomy in patients with seizures secondary to cavernous malformations. Clin. Neurosurg. 55, 101-107.

Kwan, P., Arzimanoglou, A., Berg, A. T., Brodie, M. J., Allen Hauser, W., Mathern, G., Moshe, S. L., Perucca, E., Wiebe, S., and French, J. (2010). Definition of drug resistant epilepsy: consensus proposal by the ad hoc task force of the ILAE Commission on Therapeutic Strategies. Epilepsia 51, 1069-1077.

Mccormick, W. F. (1966). The pathology of vascular ("arteriovenous") malformations. J. Neurosurg. 24, 807-816.

Menzler, K., Chen, X., Thiel, P., IwinskaZelder, J., Miller, D., Reuss, A., Hamer, H. M., Reis, J., Pagenstecher, A., Knake, S., Bertalanffy, H., Rosenow, F., and Sure, U. (2010). Epileptogenicity of cavernomas depends on (archi) cortical localization. Neurosurgery 67, 918-924.

Moran, N. F., Fish, D. R., Kitchen, N., Shorvon, S., Kendall, B. E., and Stevens, J. M. (1999). Supratentorial cavernous haemangiomas and epilepsy: a review of the literature and case series. J. Neurol. Neurosurg. Psychiatr. 66, 561-568.

Morrell, F. (1985). Secondary epileptogenesis in man. Arch. Neurol. 42, 318-335.

Morrell, F. (1991). The role of secondary epileptogenesis in human epilepsy. Arch. Neurol. 48, 1221-1224.

Otten, P., Pizzolato, G. P., Rilliet, B., and Berney, J. (1989). 131 cases of cavernous angioma (cavernomas) of the CNS, discovered by retrospective analysis of 24,535 autopsies. Neurochirurgie 35, 82-83, 128-131.

Piepgras, D. G., Sundt, T. M. Jr., Ragoowansi, A. T., and Stevens, L. (1993). Seizure outcome in patients with surgically treated cerebral arteriovenous malformations. J. Neurosurg. 78, 5-11.
Pollock, B. E. (2008). Radiosurgery for cavernous malformations: theory and practice. Clin. Neurosurg. 55 97-100.

Pozzati, E., Giuliani, G., Nuzzo, G., and Poppi, M. (1989). The growth of cerebral cavernous angiomas. $\mathrm{Neu}$ rosurgery 25, 92-97.

Raychaudhuri, R., Batjer, H. H., and Awad, I. A. (2005). Intracranial cavernous angioma: a practical review of clinical and biological aspects. Surg. Neurol. 63, 319-328.

Regis, J., Bartolomei, F., Kida, Y. Kobayashi, T., Vladyka, V., Liscak, R., Forster, D., Kemeny, A., Schrottner, O., and Pendl, G. (2000). Radiosurgery for epilepsy associated with cavernous malformation: retrospective study in 49 patients. Neurosurgery 47, 1091-1097.

Revencu, N., and Vikkula, M. (2006). Cerebral cavernous malformation: new molecular and clinical insights. J. Med. Genet. 43, 716-721.

Robinson, J. R., Awad, I. A., and Little, J. R. (1991). Natural history of the cavernous angioma. J. Neurosurg. 75 , 709-714.

Schneider, B. F., Eberhard, D. A., and Steiner, L. E. (1997). Histopathology of arteriovenous malformations after gamma knife radiosurgery. $J$. Neurosurg. 87, 352-357.

Schroeder, H. W., Gaab, M. R., and Runge, U. (1997). Supratentorial cavernous angiomas and epileptic seizures: preoperative course and postoperative outcome. Neurosurgery 40, 885.

Siegel, A. M., Roberts, D. W., Harbaugh, R. E., and Williamson, P. D. (2000). Pure lesionectomy versus tailored epilepsy surgery in treatment of cavernous malformations presenting with epilepsy. Neurosurg. Rev. 23, 80-83.

Stavrou, I., Baumgartner, C., Frischer, J. M., Trattnig, S., and Knosp, E. (2008). Long-term seizure control after resection of supratentorial cavernomas: a retrospective singlecenter study in 53 patients. Neurosurgery 63, 888-896; discussion 897.

Stefan, H., and Hammen, T. (2004). Cavernous haemangiomas, epilepsy and treatment strategies. Acta $\mathrm{Neu}$ rol. Scand. 110, 393-397.

Stefan, H., Scheler, G., Hummel, C. Walter, J., Romstock, J., Buchfelder, M., and Blumcke, I. (2004). Magnetoencephalography (MEG) predicts focal epileptogenicity in cavernomas. J. Neurol. Neurosurg. Psychiatr. 75, 1309-1313.

Sugano, H., Shimizu, H., and Sunaga S. (2007). Efficacy of intraoperative electrocorticography for assessing seizure outcomes in intractable epilepsy patients with temporallobe-mass lesions. Seizure 16, 120-127.

Van Donselaar, C. A., Schimsheimer, R. J., Geerts, A. T., and Declerck, A C. (1992). Value of the electroencephalogram in adult patients with untreated idiopathic first seizures. Arch. Neurol. 49, 231-237.

Van Gompel, J. J., Marsh, W. R., Meyer, F. B., and Worrell, G. A. (2010). Patient-assessed satisfaction and outcome after microsurgical resection of cavernomas causing epilepsy. Neurosurg. Focus 29, E16.

Van Gompel, J. J., Rubio, J., Cascino, G. D., Worrell, G. A., and Meyer, F. B. (2009). Electrocorticographyguided resection of temporal cavernoma: is electrocorticography warranted and does it alter the surgical approach? J. Neurosurg. 110, 1179-1185.

Von Der Brelie, C., and Schramm, J. (2011). Cerebral cavernous malformations and intractable epilepsy: the limited usefulness of current literature. Acta Neurochir. (Wien) 153, 249-259.

Wieser, H. G., Blume, W. T., Fish, D., Goldensohn, E., Hufnagel, A., King, D., Sperling, M. R., Luders, H., and Pedley, T. A. (2001). ILAE Commission Report. Proposal for a new classification of outcome with respect to epileptic seizures following epilepsy surgery. Epilepsia 42, 282-286.

Williamson, A., Patrylo, P. R., Lee, S., and Spencer, D. D. (2003). Physiology of human cortical neurons adjacent to cavernous malformations and tumors. Epilepsia 44, 1413-1419.

Yeh, H. S., and Privitera, M. D. (1991). Secondary epileptogenesis in cerebral arteriovenous malformations. Arch. Neurol. 48, 1122-1124.

Yeh, H. S., Tew, J. M. Jr., and Gartner, M. (1993). Seizure control after surgery on cerebral arteriovenous malformations. J. Neurosurg. 78, 12-18.

Yeon, J. Y., Kim, J. S., Choi, S. J., Seo, D. W., Hong, S. B., and Hong, S. C. (2009). Supratentorial cavernous angiomas presenting with seizures: surgical outcomes in 60 consecutive patients. Seizure 18, 14-20.

Zabramski, J. M., Wascher, T. M., Spetzler, R. F., Johnson, B., Golfinos, J., Drayer, B. P., Brown, B., Rigamonti, D., and Brown, G. (1994). The natural history of familial cavernous malformations: results of an ongoing study. J. Neurosurg. 80, 422-432.

Zevgaridis, D., Van Velthoven, V., Ebeling, U., and Reulen, H. J. (1996). 
Seizure control following surgery in supratentorial cavernous malformations: a retrospective study in 77 patients. Acta Neurochir. (Wien) 138, 672-677.

Conflict of Interest Statement: The authors declare that the research was conducted in the absence of any commercial or financial relationships that could be construed as a potential conflict of interest.

Received: 18 August 2011; paper pending published: 29 September 2011; accepted: 07 December 2011; published online: 03 January 2012.
Citation: Alonso-Vanegas MA, CisnerosFranco JM and Otsuki T (2012) Surgical management of cavernous malformations presenting with drugresistant epilepsy. Front. Neur. 2:86. doi: 10.3389/fneur.2011.00086

This article was submitted to Frontiers in Epilepsy, a specialty of Frontiers in Neurology.
Copyright (C) 2012 Alonso-Vanegas, Cisneros-Franco and Otsuki. This is an open-access article distributed under the terms of the Creative Commons Attribution Non Commercial License, which permits non-commercial use, distribution, and reproduction in other forums, provided the original authors and source are credited. 